\title{
A review of map types used in empirical research in cartography
}

\author{
Katarzyna Słomska ${ }^{a}$ * \\ ${ }^{a}$ University of Warsaw, Faculty of Geography and Regional Studies, Chair of Geomatics and Information Systems, \\ k.slomska@uw.edu.pl \\ * Corresponding author
}

Keywords: empirical research, map stimuli, reporting studies

\begin{abstract}
:
The main aim of this paper is to present types of maps used as stimuli in empirical studies in cartography in the context of the user and researcher perspective. Such revisions could possibly bring opportunities similarly to the taxonomy of interaction primitives proposed by Roth (2013) or data base focused on studies' participants by White (2017). Their work is a systematisation of previous knowledge, it constitutes a framework for future studies and makes them easier to compare and validate.

It was decided to focus on papers published in years 2000-2017. The query covered journals affiliated to the ICA: The Cartographic Journal, Cartographica: The International Journal for Geographic Information and Geovisualization, and Cartography and Geographic Information Science, and the journal published by the ICA: the International Journal of Cartography. From the wide scope of geovisualisations used in empirical studies only maps were included.

By elaboration of a matrix of map types, which juxtaposed maps according to objective criteria, and performance of a meta-analysis of the gathered data, the extensive group of studies can be more precisely characterised. The review juxtaposed 103 research papers presenting an experiment where a map was used as the stimulus. They constituted $8.6 \%$ of papers published in the mentioned journals since 2000. In the analysed period the number of research papers concerning empirical studies in cartography has risen (Figure 1). Unfortunately, it is not possible to specify the number of maps used as stimuli in the analysed papers, as authors do not always provide this information. However, organised description of the stimuli would provide an opportunity for repetition and, consequently, verification of results. The matrix is available on-line with the accompanying paper (doi.org/10.2478/mgrsd-2018-0014).
\end{abstract}

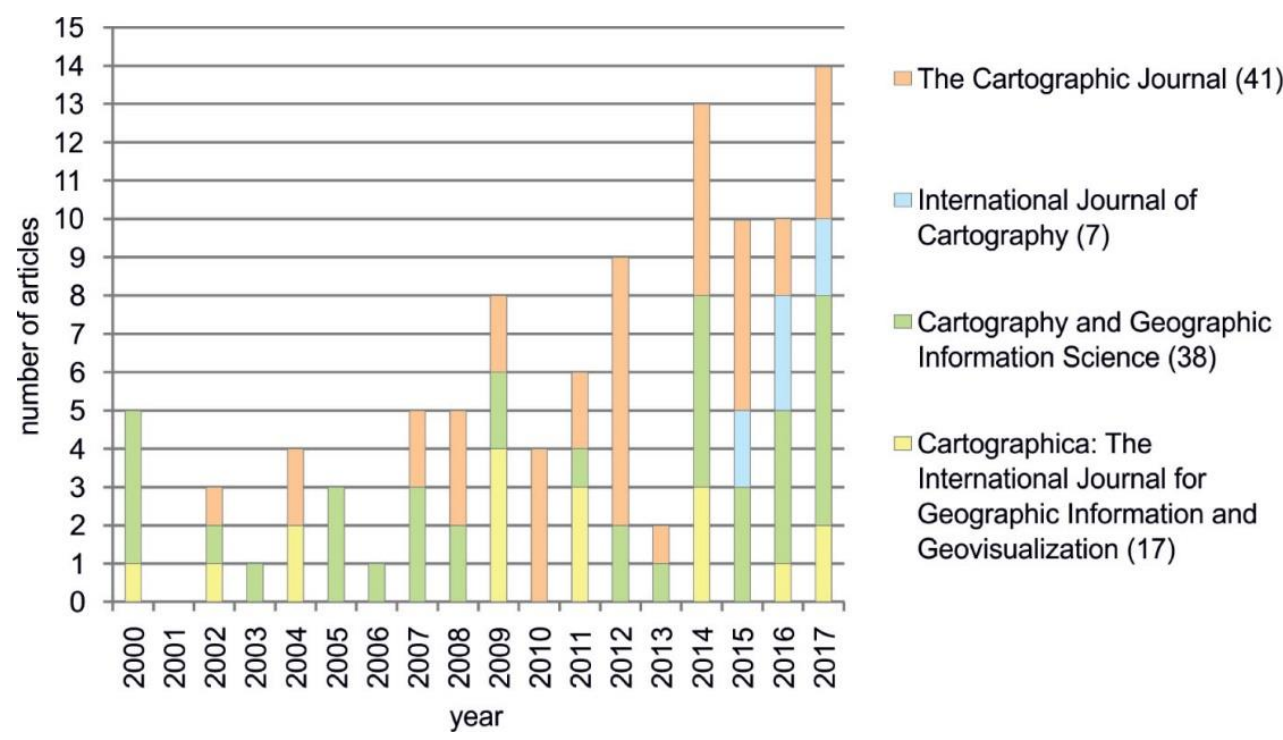

Figure 1. Number of analysed papers per year

The attributes of maps used for the identification of map types were chosen on the basis of the author's description of stimuli. Four main features were chosen (Figure 2): map medium (paper, screen), reactiveness (interactive, noninteractive), method of cartographic presentation (quantitative, qualitative), users' familiarity with the presented area and data (familiar, unfamiliar, fictional). In most experiments more than one type of map was used. Such experiments are presented in figure 2 as transitional areas, marked by vertical banding in the middle of the figure, in order to point out differences in overlapping proportions between features. 


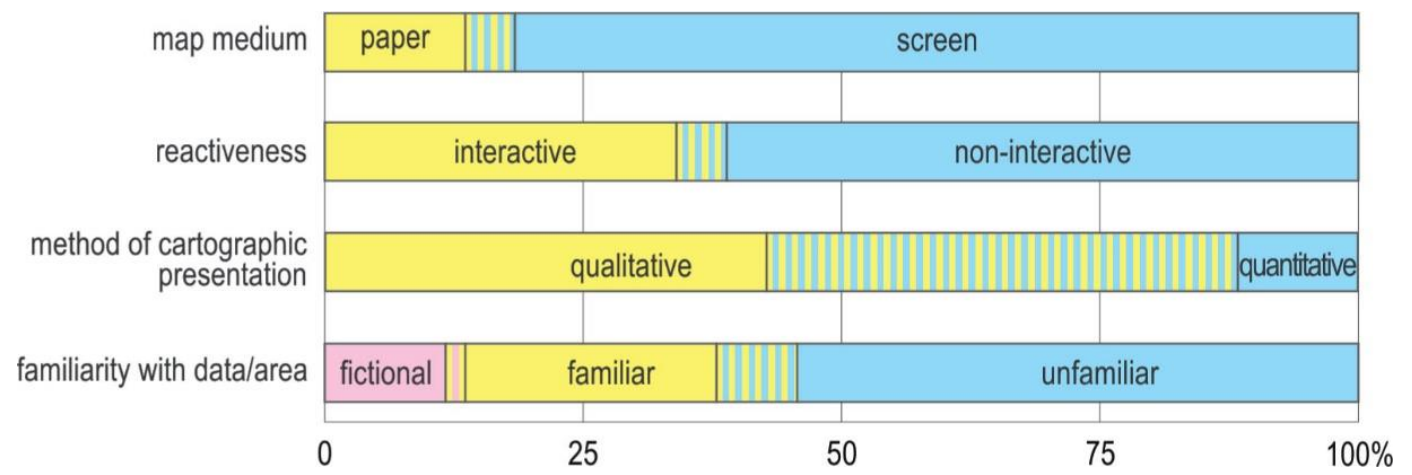

Figure 2. Percent of papers in which particular types of maps were used

The predominance of digital maps in the whole analysed period could be observed. With rising availability of geoportals, one could predict the increase of interactive maps use. Nonetheless, they were used in less than a half studies. What may be also thought-provoking is that the percentage of paper maps used in experiments have risen in last years. It shows that both types of media are thought to be equally important.

In the scope of every analysed feature, one of the types could be observed to dominate (Figure 2). The most common types are maps displayed on a screen (88\%), non-interactive (66\%), qualitative (88\%) and unfamiliar (62\%). Studies that applied maps that fall into all these dominant categories simultaneously were the subject of $25 \%$ of all experimental studies.

The elaboration of a consistent database of map types is a continuation of the work by Roth (2013) and White (2017) and another step towards enhancing the reporting of empirical studies in cartography. Complying with this postulate would allow studies to be better validated and compared. In order to apply this proposal, explicit characteristic were defined. This allows this database to be further developed. Furthermore, the described database could be integrated with the database concerning participants by White (2017), which would allow profound insight to be gained into the empirical research conducted by cartographers since 2000 .

By juxtaposing maps according to objective criteria and performing a meta-analysis of the gathered data, the extensive group of studies can be more precisely characterised. The conclusions may serve other researchers who would like to make informed decisions when preparing their studies. In summary, meticulous description of experimental studies is crucial for proper construction of forthcoming research. Revisions of conducted experiments may constitute a frame for reporting study results.

\section{References}

Roth, RE 2013, 'Cartographic Interaction Primitives. Framework and Synthesis', The Cartographic Journal, vol. 49, no. 4, pp. 376-395.

White, T 2017, 'Identifying Best Practices by Examining Relationships Between Reporting in User Studies and Design of User Studies'. ICC 2017 Proceedings.

This work was supported by the Faculty of Geography and Regional Studies at the University of Warsaw. 